\title{
A New Approach on the System-Level Design for the Wireless Sensor Network
}

\author{
HU Bowen ${ }^{1, \text { a }}$ \\ ${ }^{1}$ School of Electronics and Information Engineering, Jingchu University of Technology, Jingmen \\ 448000, China \\ a hubowen@qq.com
}

Keywords: Wireless Sensor Network; System-Level Design; Hierarchical Heterogeneity

\begin{abstract}
With sensor technology matures, wireless sensor networks in the field more and more attention and has been widely applied. As the wireless sensor network system behavior is heterogeneous, traditional wireless sensor network system design modeling focuses on single subsystem model behavior from the wireless sensor network system modeling and simulation. System-level design methodologies have been introduced in the wireless sensor network modeling and simulation methodology, system-level design methodology wireless sensor networks as a complete system, in order to view the embedded system wireless sensor network system is divided into various independent system behavior The Unified Modeling module, to make up for the shortcomings of traditional single wireless sensor network system modeling, the study of wireless sensor network system level design methodology, in a heterogeneous environment of wireless sensor network system modeling and simulation has important theoretical and practical significance.
\end{abstract}

\section{Introduction}

As CMOS technology advances, more and more small circuit boards, a lot of electronic equipment installed in the radio subsystem, greatly promoted the development of wireless sensor technology. These miniature sensors can perform a variety of sensing tasks, including sound and seismic monitoring survey [1-2]. They can help people collect data in remote areas or unsuitable for human survey area. Wireless sensor network system has been used in various fields, vehicles can use the wireless micro-sensor control engine status, tire pressure, and adjust the gasoline standards; production lines of these sensors can also be used to manipulate production steps; in the military environment, miniature sensors can also be from thrown down to the ground on the plane to track the target activities [3].

Due to the widespread use of wireless sensor network system, so that for modeling and simulation of wireless sensor network system becomes very important. However, high dynamic and multichannel communication system makes wireless sensor network modeling and simulation of wireless sensor network system model is very complex. Meanwhile, the wireless sensor network system synchronization for real-time interaction, concurrency, reliability and time requirements are high, the urgent need for a robust design methods to guide the design, to solve their complex problems [4]. Because a complete wireless sensor network system includes a variety of different systems and different behavioral factors, and traditional modeling and simulation platforms are based on a single frame, it cannot subsystem wireless sensor network system in different behaviors effective coupling, so the traditional wireless sensor network system design methods to separate the system into subsystems different behavior, but unilateral conduct simulation studies from the system, such as focusing on the modeling analysis, or top-level application of the underlying protocol, not from the global overall to consider the interaction between the various subsystems of wireless sensor network system, so that the design of wireless sensor network system is very complex. Therefore, an effective modeling and simulation design methodology for the design of the sensor network system has a very important theoretical significance. 


\section{Wireless sensor network system architecture}

Wireless sensor network system network architecture shown in Figure 1, the wireless sensor network systems often include distributed sensor nodes (group), sink node (gateway node), the Internet and the user interface [5-6]. A large number of sensor nodes are randomly distributed in the monitoring area, the network can be constructed by self-organization. Each sensor node will own the monitored useful information after preliminary data processing and information fusion, along other sensor nodes transmit hop, monitoring information and data during transmission may be more than one node processing, through multiple hops After the route to the sink node, and then through the sink node to a satellite or cable network connections transferred to the end user.

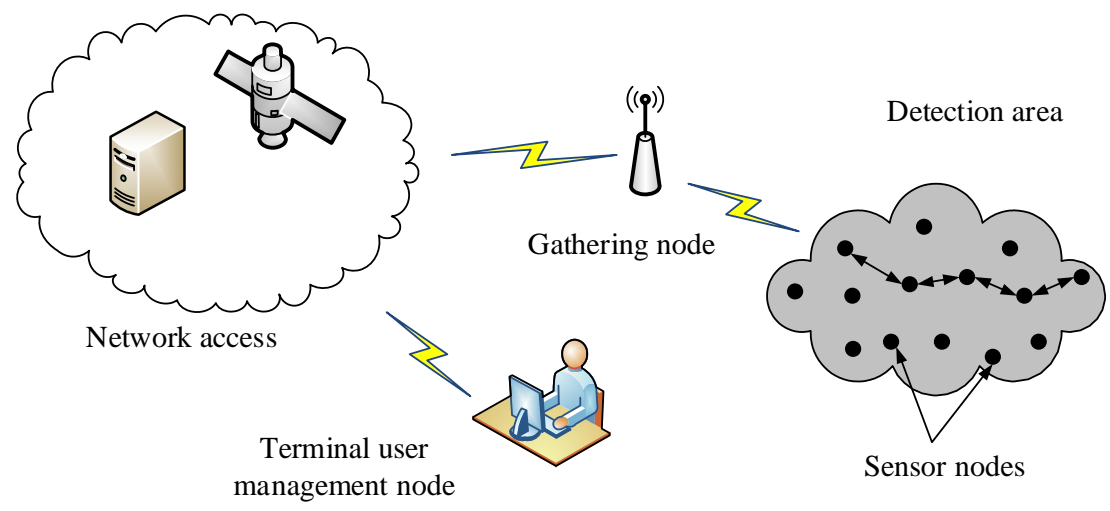

Figure 1.Architecture of wireless sensor network system

Sensor nodes are usually a miniature embedded systems [7-8], its processing power, storage capacity and communication capacity is relatively weak, unable to carry the battery is replaced. Judging from the network function, each sensor node both terminal and routing functionality of traditional network nodes, in addition to information collection and data processing, but also forwarded data and other storage, management and integration of the processing of other sensor nodes, while the Other nodes collaborate on specific tasks. Sink node processing power, storage capacity and communication capacity is relatively strong, its connection to external networks, wireless sensor networks and the Internet, to achieve communication protocol conversion between the two protocols, as well as publish application node monitoring tasks, and to gather information data is forwarded to the external network. Sink node can be either a sensor node with enhanced features, there is enough energy supply and more storage space, it can also be no monitoring function only with a special gateway device wireless communication interface.

Composition of wireless sensor network node is different depending on the application, a typical sensor nodes are usually by the sensor unit, a processor unit, a wireless communication unit and power supply unit composed of four parts, shown in Figure 2. The sensor unit is responsible for monitoring the regional collection and data conversion information, the form of monitoring of the physical information determines the type of sensor unit; a processor unit, the control operation of the entire sensor nodes, storage and processing of data, and external data acquisition itself; wireless communication unit through a radio channel sensor nodes communicate with other, exchange control messages and data; power supply unit provides the required energy for the sensor node runs.

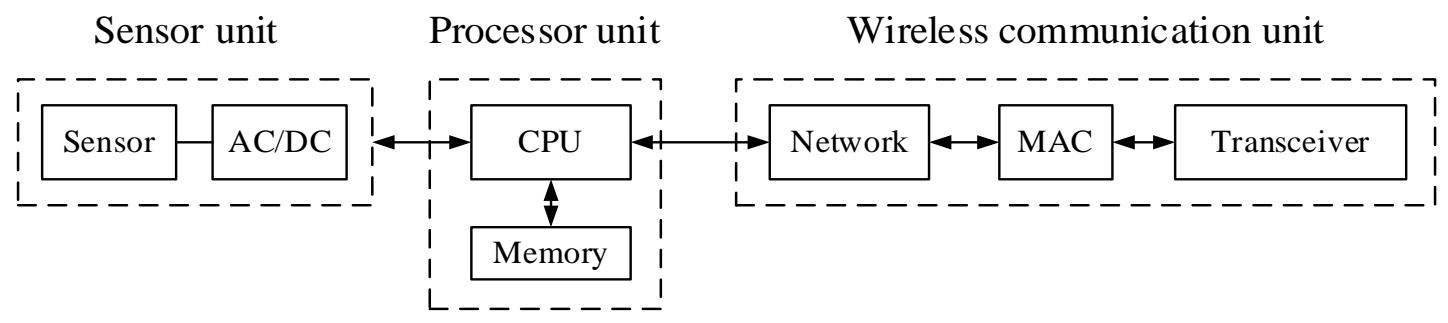

Figure 2. Wireless sensor network node component unit 


\section{System-level modeling of hierarchical heterogeneity}

The idea is to hierarchical heterogeneity into a number of different subsystems behavior, how the system is divided into a number of workable and clear calculation model subsystems become one of the most important issues. Component-based design school advocate the system is decomposed into a number of well-defined subsystems, and each subsystem has a different function. Object-oriented design methodology object abstraction, through the class hierarchy and function calls and other methods to the design of complex systems. This design approach often used in the development and design of real-time software, emphasizing the use of UML language to formally describe the system; for middleware design methodology will promote one or more objects to encapsulate the concept of service, and then a combination of these services into the system, because the design of science-oriented middleware, the target object need to work together to provide the required logic functions. Although object-oriented design methodology and design methodology oriented middleware logical distinction, but their basic structure of the system components are interconnected objects, and interfaces between objects are linked to a function call, the program calls the function directly. The control data flow from one object to another. The system features are hidden in the function call interface. Therefore, object-oriented design methodologies and middleware-oriented design methodology is emphasized how the system is broken down into various subsystems, and no combinations include subsystems.

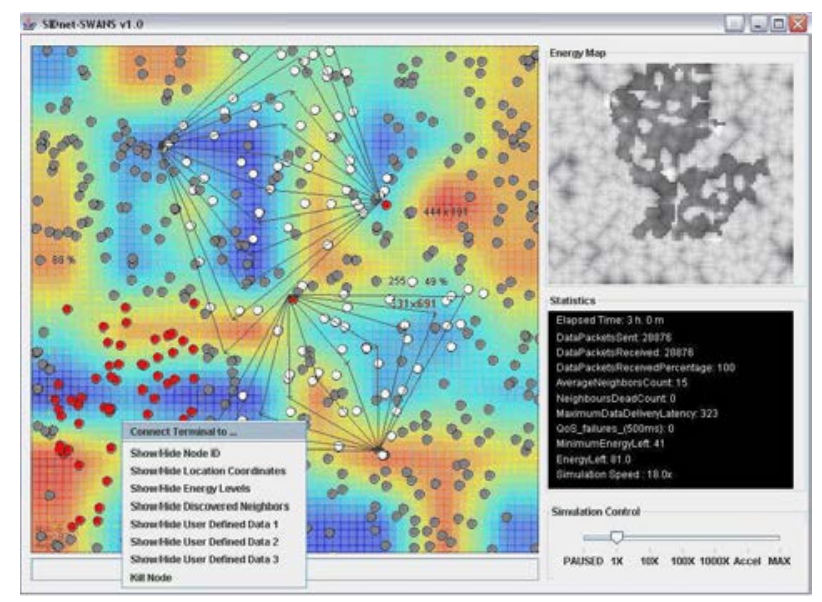

Figure 3. Case for Wireless Sensor Network Simulator

Ptolemy level heterogeneous use role-oriented system-level design methodology, based on system-level description of the behavior of Ptolemy II wireless sensor networks targeting system, this paper can put the whole model for wireless sensor networks targeting system is divided into three modules (tested targets, sensors, positioning module), respectively modeling, and can be simulated, as shown in Figure 3.

\section{Wireless sensor network system level design}

The entire wireless sensor network positioning system-level model consists of four major components: a test sound goal, a number of sound sensor nodes, a number of sink nodes (location node) and two wireless information channel. The network design process shown in Figure 4.

In the initial stage of the experiment is running, you need to initialize the network topology, the system uses a random function of the role of a global control for all of the analog sensor nodes within the framework of the random distribution of position, in order to simulate the random behavior sensor arrangement scene; sink node (ie location node) is determined using a single relay of communication, when a node receives an adjacent node information from three different when it is defined as a sink node, while the open positioning. Sensor nodes are converted into sound sensor, this step is by creating a sound input port node model completed. Because the use of the type of channel depends on the port channel selection attribute identity, so the sound sensor ports must be subject to control the sound channel. Task sound sensor node model is the effective detection range, 
and listens for the sound information from the sound channel. Upon receipt of the valid information, record information reception time, then the node's own position information and time information is forwarded to the radio channel through the output port.

Responsible for two wireless information channel communications, are responsible for the spread of the sound information and wireless information, using the Limit Range Channel role, its properties can be divided into reliable transport behavior / unreliable transmission characteristics depending on the application, limited transmission range / unlimited transmission range, limited transfer rate / unlimited transfer rate.

The core of the wireless sensor network targeting system - positioning node, using triangulation algorithm to determine the coordinates of the target position measured voice. The node has a wireless input port via data radio channel sound output port wireless sensor nodes, and ultimately determine the position of the measured sound objective. The positioning node must have at least from three different sound sensor node information in order to use triangulation algorithms.

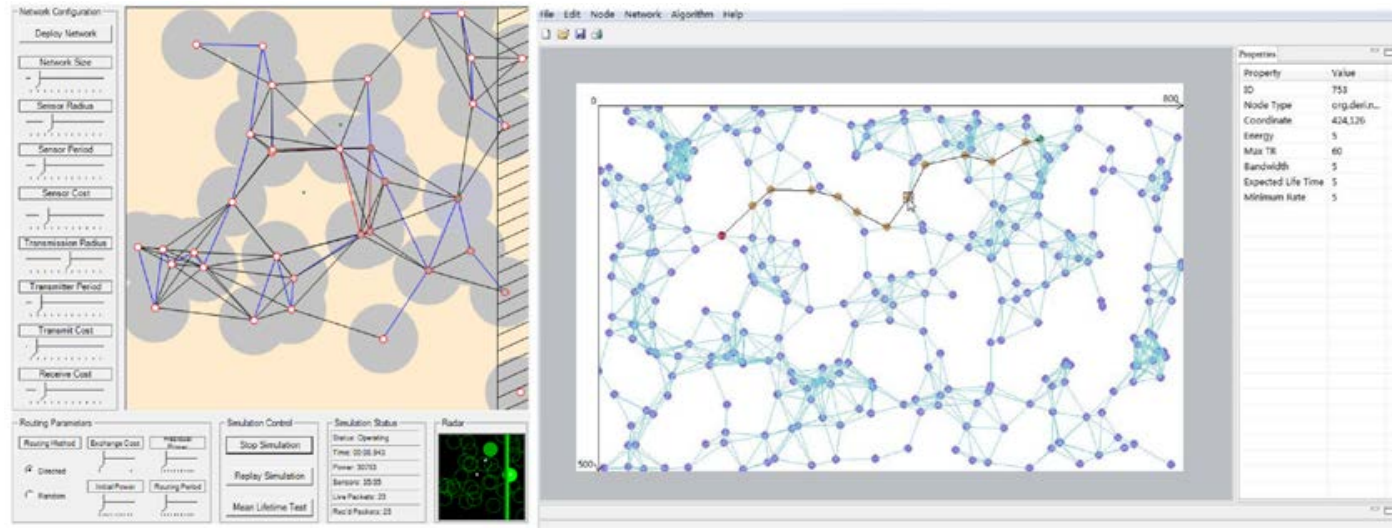

Figure 4. The wireless sensor network by system level process

\section{Conclusion}

Since conventional modeling and simulation environment based on a single framework for modeling, so it can not be the wireless sensor network system all the various factors into account; a new design methodology - system-level design methodologies have been introduced in the modeling and simulation of wireless sensor networks methodology, system-level design methodology wireless sensor networks as a complete system, in order to view the embedded system wireless sensor network system is divided into separate modules for each system behavior unified modeling, make up the traditional wireless sensor network system The disadvantage of a single model, Based on this methodology, the wireless sensor network system in more depth and design of modeling and simulation.

\section{Reference}

[1] Huang Y M, Hsieh M Y, Chao $\mathrm{H} \mathrm{C}$, et al. Pervasive, secure access to a hierarchical sensor-based healthcare monitoring architecture in wireless heterogeneous networks[J]. Selected Areas in Communications, IEEE Journal on, 2009, 27(4): 400-411.

[2] Ceriotti M, Mottola L, Picco G P, et al. Monitoring heritage buildings with wireless sensor networks: The Torre Aquila deployment[C]/Proceedings of the 2009 International Conference on Information Processing in Sensor Networks. IEEE Computer Society, 2009: 277-288.

[3] Shin S, Kwon T, Jo G Y, et al. An experimental study of hierarchical intrusion detection for wireless industrial sensor networks[J]. Industrial Informatics, IEEE Transactions on, 2010, 6(4): 744-757.

[4] Rabaey J. Low power design essentials[M]. Springer Science \& Business Media, 2009. 
[5] Jardosh S, Ranjan P. A survey: Topology control for wireless sensor networks[C]//Signal Processing, Communications and Networking, 2008. ICSCN'08. International Conference on. IEEE, 2008: 422-427.

[6] Wan Y, Li L, He J, et al. Anshan: Wireless sensor networks for equipment fault diagnosis in the process industry[C]//Sensor, Mesh and Ad Hoc Communications and Networks, 2008. SECON'08. 5th Annual IEEE Communications Society Conference on. IEEE, 2008: 314-322.

[7] Islam M M, Hassan M M, Lee G W, et al. A survey on virtualization of wireless sensor networks[J]. Sensors, 2012, 12(2): 2175-2207.

[8] Celandroni N, Ferro E, Gotta A, et al. A survey of architectures and scenarios in satellite based wireless sensor networks: system design aspects[J]. International Journal of Satellite Communications and Networking, 2013, 31(1): 1-38. 